\title{
Folk Music of the Masterpiece of Han Folk, Tibetan Folk and Qiang Tribe--The New Artistic Aesthetic Value of Sichuan Xiling Mountain Chants
}

\author{
Yuanchuan Pan \\ Normal College of Shenzhen University, Guangdong Shenzhen 518000, China. \\ ychpan@163.com.
}

\begin{abstract}
The national intangible cultural heritage, Xiling mountain chants, have been spreaded for hundreds of years and unfailing, in the multiethnic area of Xiling Snow Mountain of Sichuan, where Han folk, Tibetan folk and Qiang tribe people lived, showed strong vitality. Xiling mountain chants are integrated multi-ethnic cultural characteristics of Han, Tibetan and Qiang nationalities, both eclecticism and self-contained, embodied unique artistic charm. This article will analyze the music features of the multiethnic Xiling mountain chants from the angle of the Anthropology of Music, as well as discuss the music style and art characteristics, and reveal the unique music art culture of the Xiling mountain chants.
\end{abstract}

Keywords: Xiling mountain chants; intangible cultural heritage; music form; aesthetic value of art.

\section{汉藏羌民族音乐的千古绝唱---四川西岭山歌艺术审美价值新解}

\section{潘原钏}

深圳大学师范学院, 广东深圳 518000 , 中国

摘 要: 国家级非物质文化遗产-----西岭山歌, 在四川西岭雪山汉藏㒸多民族聚居地区流传 了数百年, 经久不衰, 展现了西岭山歌顽强的生命力。西岭山歌融合了汉藏美多民族的文化 特点, 既兼收并蓄, 又自成体系, 体现了西岭山歌独特的艺术魅力。现从音乐人类学角度, 分析汉藏羌多元一体的音乐文化形态, 探讨西岭山歌的音乐风格和艺术特征, 揭示西岭山歌 独特的音乐艺术文化。

关键词：西岭山歌；非物质文化遗产; 音乐形态; 艺术审美价值 (关键词为4 7个)

\section{1. 前言}

关于四川西岭山歌的研究, 也是近十几年的事情, 即从 2004 年西岭山歌传人张道深老人带领 当地村民，对濒临失传的西岭山歌的挖掘、采集和整理开始，在当地政府、西岭山歌协会、 民间艺人的共同努力下, 逐步把西岭山歌引进学校, 带进旅游景区, 将西岭山歌逐步打造成 了大邑县 “文旅兴县” 的品牌, 打造成了国家级非物质文化遗产, 对推动西岭山歌的传承发 展起到了积极的作用。除此之外, 国内缺乏对西岭山歌的理论研究, 尤其缺乏从音乐理论角 度的深度研究, 在国内关于西岭山歌的理论文章和学术论文很少, 寥察无几。鉴于此, 为弘 扬中国民间音乐, 笔者从 2012 年开始, 以音乐人的使命感走进西岭雪山, 通过广泛走访西岭 山歌传承人及当地民间艺人, 潜心研究, 发现了西岭山歌的音乐之美, 因此, 本文结合几年 来的研究成果, 从音乐理论的专业角度, 分析西岭山歌的音乐形态及艺术特征, 探讨西岭山 歌的艺术价值, 揭示西岭山歌独特的音乐艺术文化, 更好地推动西岭山歌的传承和发展。 


\section{2. 西岭山歌的起源与分类}

唐代诗人杜甫曾作七言绝句诗形容西岭大山的雪景：“两个黄婯鸣翠柳，一行白鹭上青天。 窗含西岭千秋雪, 门泊东吴万里船。”西岭雪山不仅有迷人的雪景, 更有美丽的西岭山歌, 汉藏差多民族融合的民族音乐文化瑰宝。虽几经沉浮，但在新的历史条件下，又在西岭雪山 深处广为传唱, 焕发出了勃勃生机。

2.1. 西岭山歌的起源

西岭山歌属于西南高原多民族风格色彩区民族音乐，相传产生于明末清初，发源并流传于四 川省大邑县㒸、汉、藏民族聚居的西岭雪山高原地区，山歌的传播以西岭镇为主，分布于花 水湾、出江镇以及芦山县的大川镇等地。西岭山歌盛行于清代、民国时期至上世纪五十年代, 如今已经口口相传了数百年, 既保留了汉族音乐的调式调性色彩, 又融合了藏族、芫族文化 特点, 既体现了西岭山歌对汉文化的历史继承, 又体现了西岭山歌汉藏差多元一体的音乐文 化特征。兼收并蓄, 自称体系。然而, 西岭山歌在近几十年的传承发展中几乎断代了, 尤其 是随着一些老一辈传承人相继离世, 大量优秀的西岭山歌曲牌以及一些独特的演唱方式和方 法濒临失传。

2004 年, 西岭人重拾西岭山歌, 对濒临失传的西岭山歌加强挖掘、采集和整理, 通过山歌协 会，民间艺人，在政府的支持下，又逐步发展起来。为了拯救和振兴西岭山歌，2007 年 5 月 成立了西岭山歌协会，西岭山歌协会和大邑县文化宣传部共同举办的西岭山歌节以及山歌歌 唱比赛，带动了当地的乡村旅游，使西岭山歌成为大邑县 “文旅兴县” 战略的重要部分。2008 年成都市人民政府将西岭山歌纳入 “成都市第二批市级非物质文化遗产” 名单中。2011 年西 岭山歌入围四川省第三批非物质文化遗产名录, 并于 2014 年 11 月获国务院批准列入第四批 国家级非物质文化遗产名录。

2.2. 西岭山歌的分类及其特点

“西岭山歌” 是西岭雪山地区所有歌谣的统称，依其唱词内容和主题可分为劳动歌、酒歌、 山歌、情歌、风俗歌和仪式歌六大类, 唱词结构以七字四句体为基础, 并依据唱腔需要在主 体唱词之外自由即兴地加入祄字。西岭山歌的演唱形式十分多样，以独唱为主，另有领唱、 齐唱和对唱等多种形式。

2.2.1劳动歌

是西岭山歌中历史最悠久的体裁，其产生时期可追溯至明代。最具有代表性的是 “号子” 系 列，如拉木、打夯、纤夫等号子。

西岭山民在农忙、放牧、挖药、伐木等生产劳作中用劳动歌提高工作热情、传达情感。劳动 歌的节奏明快，唱词中包含较多祄词。

\subsection{2酒歌}

是西岭山歌中最能体现四川酒文化的歌曲种类，受到㒸族文化的影响，西岭山民生活中的婚 丧嫁娶、喜庆节日、请客迎宾等各种喜庆场合都离不开喝酒，喝酒时轮流唱酒歌，内容多是 祝贺、赞颂和酬谢。酒歌大多音调高立, 但音域不宽, 节奏较为缓慢, 有齐唱、对唱、独唱 等表现形式。

\subsection{3山歌与情歌}

山歌是西岭山歌中运用最为广泛的歌曲种类, 多即兴演唱, 音域较宽, 曲调嘹亮而高立, 节 奏较为自由, 以独唱形式为主。表达男女爱慕之情的山歌尤为丰富, 爱幕、追求、热恋、相 思等均可用山歌来表达。情歌词意诙谐逗趣, 咏唱爱慕、恋爱、求婚、夫妻情、反抗封建婚 姻、甚至是与偷情相关的主题也出现在西岭山歌的情歌之中。

2.2.4 风俗歌及仪式歌

则在婚丧和祭山仪式中表现得最为充分。如受㒸族民俗文化的影响, 西岭曾有每年五月初五 举行祭山的传统。不仅祭山时要唱仪式歌, 祭山后走村串寨, 挨家挨户唱风俗歌和仪式歌也 是当地重要习俗之一。 


\section{3. 西岭山歌音乐形态与唱词特征分析}

笔者通过多次田野考察发现, 西岭人并不以唱词题材将西岭山歌进行分类, 而是更为重视 “调 门” 的选择。“调门” 是特定的旋律组织, 西岭山歌歌谣体系包含老调、大调、小调、吆噢 调、呀妹调等五种原生态调门。演唱者在选择调门之后即兴编配不同内容的歌词, 从而形成 劳动歌、酒歌等不同类型的民歌。在演唱实践中, 西岭人视第一句歌词为曲名, 通常会在演 唱前告知听众调门名称和曲名, 如: 吆噢调——唱起山歌有精神。

3.1 吆噢调

下面以吆噢调——《唱起山歌有精神》为例, 分析西岭山歌的音乐形态与唱词特征 吆噢调的唱词为七字四句体, 在小十度的音区中呈现羽调式的旋律进行。风格奔放、粗旷, 曲调嘹亮, 第一句收尾处呈现铿铭有力的唱腔结合 “哟嗬嗬 ” 祄字组合是吆噢调的主要特 点之一。吆噢调各句的句间和句尾均采用祄词结合较长的拖腔, 体现出㒸族高腔型山歌的特 点。

在旋律进行和节奏安排方面, 第一句分为三个部分, 每一部分均以长音收腔。前两个部分演 唱七字主体歌词, 开头音调高昂, 多次出现旋律最高音, 以祄字 “哟” 配合长音收尾。第三 部分则以强而有力的 “哟嗬嗬” 祄字组合结合下行构成的三音音型, 使吆噢调体现出山歌特 有的豪迈精神。

第二句至第四句各分为两部分, 以伴有祄词的长音为界。第二句句中以祄字 “吆” 演唱的下 行级进加小三度音型和长音与第一句结尾形成相互辉映。第二句最终以整首歌曲的最低音收 尾。第三句第一部分有别于第二句的抒情风格, 完全以三度、四度跳进起伏的方式进行。值 得一提的是, 第三句句中的祄字 “噻 (se) ” 是四川方言中特有的感叹词, 经常用于日常对 话中, 如: 是噻。演唱中运用 “噻” 字不仅体现出鲜明的地方色彩, 更为山歌增添了朴实、 粗旷的审美情趣。在第三句结尾处首次采用祄字 “吔”，并在收尾处出现甩腔。

第四句不论是旋律进行还是唱词安排方面都与第二句近似, 为配合行腔, 在主体唱词第四字 和第五字、第六字和第七字之间以及第七字之后加入祄字, 并在第六字与第七字之间采用下 行二三度音型将旋律分为前后两段。下行二三度音型同时也是第一、二、四句的收尾音型。 较为特殊的是, 全曲的最低旋律音仅出现在第二句和第四句结尾处, 与第一句开头高六的最 高旋律音对应，使整个吆噢调呈现起高收低的旋律模式。

吆噢调的代表作品有《唱起山歌有精神》和《西岭山上沟坎多》, 描述西岭人在工作中通过 山歌演唱振奋精神和相互联系感情的情景, 赞扬西岭山歌对西岭地区人民的劳动生活的正面 影响。 


\section{吆噢调:《唱起山歌有精神》}

演唱：任汉成

记谱: 潘原钏

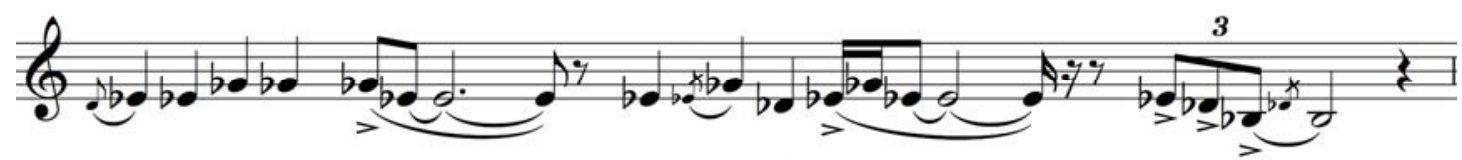

唱起山歌哟-ー - 有精 神哟-ーー - 哟㺃㺃 -

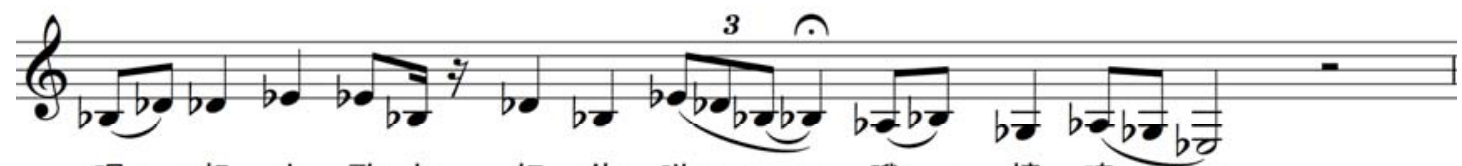

唱一起 山 歌来 好 传 哟- - - 哦 - 情 噢 - -
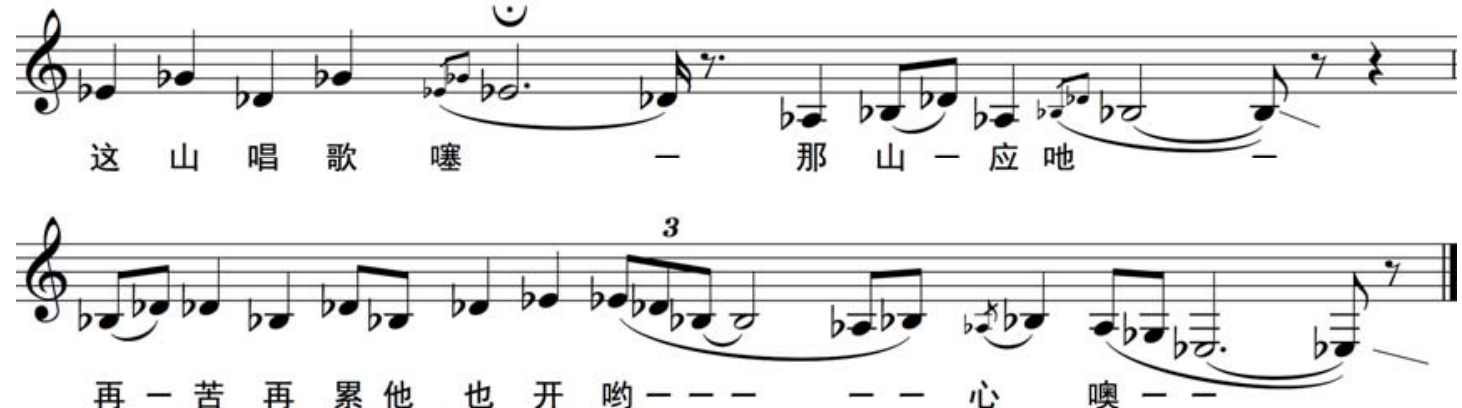

吆噢调的唱词为七字四句体, 在小十度的音区中呈现羽调式的旋律进行。风格奔放、粗旷, 曲调嘹亮，第一句收尾处呈现铿铭有力的唱腔结合 “哟嗬嗬～” 祄字组合是吆噢调的主要特 点之一。吆噢调各句的句间和句尾均采用祄词结合较长的拖腔, 体现出㒸族高腔型山歌的特 点。

3.2 西岭山歌其他原生态调门特点

呀妹调: 与吆噢调相比, 呀妹调的音域较小, 音域为大六度, 每一句的主要旋律音区均维持 在纯四度或纯五度之内, 全曲建立在商调式的基础上, 仅在个别长音前加入一个比最低旋律 音低半音的调性外音进行装饰处理。

小调: 小调与吆噢调和呀妹调在旋律和唱词结构方面均有明显的区别, 吆噢调和呀妹调含有 丰富的华彩唱腔, 小调则多为一字一音或一字二音。主体唱词虽以七字体为基础, 但个别段 落可增加至九字，衬字中感叹词较少。

大调：大调的唱词为四句体，前两句呈现七句体，后两句则可自由加入更多主体唱词。大调 的曲调建立在角调式六声音阶的基础上, 节奏较自由, 前两句具旋律性较舒缓, 后两句则呈 现明显的说唱风格, 速度加快, 近似藏族民歌中典型的前长后短、前松后紧的节奏布局。

老调：七字四句体的老调曲风抒情而高六, 曲调中大幅度的旋律起伏, 自由且富于变化的节 奏布局以及多处运用自由延长音等特点, 均与高腔型芫族山歌不谋而合。老调为羽调式, 但 各句均不以主音结束，而是以甩腔收尾，呈现山歌中典型的呼唤表现方式。

\section{4. 西岭山歌的艺术特色}

西岭山歌具有鲜明的艺术特色、地域特色, 主要体现在题材广泛、语言丰富、调式调性、唱 腔等方面。

\section{1 题材广泛，语言丰富，文学艺术性强}

4.1. 1 西岭山歌题材广泛

包括劳动歌、酒歌、山歌、情歌、风俗礼仪歌等等, 意境含蓄, 善用比兴手法, 语言生动, 修辞优美。

如老调《阳雀叫唤桂桂阳》，“阳雀叫唤（呃）桂桂（的）阳, 我（那）情哥哥（来）在有 名堂（呃）, 她最爱听我（呃）把山歌（来）唱（昂）, 句句（那）唱在心坎上（呃）。” 
这首歌利用 “阳雀叫唤” , 形象生动的比喻 “女子唱歌” , 并带双关, 用于抒发相爱之意, 相思之情, 委婉含蓄、耐人寻味。

4.1.2注重押韵与对仗

在声韵方面, 西岭山歌很讲究语言流畅, 歌曲多以七字四句体为为基本句式, 且都十分押韵, 在演唱时，按四川话押韵。

如：大调《高山顶上一座塘》，“高山（你家）顶上（你的）一座塘（哟嗬嗬）, 看到（你 家）鱼儿（哟嗬）筷子长（chang），塘住（你的）鱼儿（你的）往水长（zhang），家住（你 那) 情妹 (吆嗬) 忘情 (吆) 郎。”

在这首山歌中，“塘”、“长”、“郎” 的韵母是 “ang”，押 “ang” 音，对仗工整，韵律 明显, 唱时琅琅上口。

在西岭山歌中, 绝大多数的发音都是方言, 因此, 独特的四川话对仗成韵, 是西岭山歌有别 于其他民歌的最为鲜明的语言特色之一。

4.1.3 唱腔悠长, 衬字使用普遍

各地方言歌谣均有自己的特点, 有的音调高昂, 有的柔美婉转, 而西岭山歌则刚柔悠长。

如: 大调《山歌越唱越好听, 铜锣越打越光生（sen）》, “山歌（你家）越唱越好听（约）, 铜锣（你嘛就）越打越光生（哟嗬嗬）; 铜锣（你嘛）就打不下（哟嗬嗬）, 山歌（你的嫂 嫂）就唱不（吆嗬嗬）成。”

这首山歌是复合式结构, 是四句式为基本句型的两段式, 演唱时普通使用祄字, 唱腔悠长, 给人以绕梁之感。

广泛使用祄字, 这是西岭山歌较为突出的特点, 可以根据表达内容和意义的不同, 依据唱腔 需要在主体唱词之外自由即兴地加入衬字。

4. 1. 4歌曲内涵丰富, 善于表达思想情感

西岭山歌具有较丰富的思想内容, 善于表达人们内心深处的情感世界, 表现手法多样, 艺术 性完整，受到人民群众的喜爱。

在西岭山歌中, 很多是通过想象来表达情感, 以物抒情, 情景交融, 形象鲜明地表现主题。 如《拉木头山歌对唱》 “......, （男）这山唱歌（哟）那山应（啰嗬哦），唱起山歌（来） 好使劲（哟嗬哦），只喊号子不应油（we），只当（那）拖田的大牯（哟）牛（啰嗬哦）。

（女）哥子要听（哟）好言劝（哟嗬哦）, 清早一碗（来）荷包（哟）蛋（啰嗬哦）, 外加 蜂蜜（瑟）加猪油（we）, 白天黑夜（来）有劲（哟）头（啰嗬哦）。

（男）尖尖山（哟）陡陡崖（ai）（哟嗬哦）, 山中有位（来）活神仙（啰嗬哦）, 神仙不 是 (瑟) 七仙女 (呃)，仙姑就是（那）么妹（哟）你（哟嗬哦）。”

这首歌是通过自由想象, 思想奔放, 充分体现出山民的那种豪放和自如, 无拘无束, 是最能 体现歌曲内涵和意义的原生态艺术形式。

4.2 旋律优美, 调式调性多样, 音乐色彩鲜明突出

由于西岭山歌是融合了汉藏㒸多民族音乐元素, 同时形成过程具有其长期的历史、地理、人 文等因素, 这就决定了西岭山歌的复杂性、多样性。除题材、语言上的差异外, 更为本质的 艺术特点是调式、调性上的特色。

西岭山歌的调式及其音乐内涵都具有浓郁的地方色彩, 从现在大量的西岭民

歌来看, 它的旋律走向、旋法多以宫、商、角、徵、羽调式为主, 这五大调式构架了西岭山 歌的主题结构。旋律优美, 几乎所有曲调中都有颤音、滑音、倚音等装饰音, 因而使旋律变 得回环曲折、委婉动听。

西岭山歌曲式结构, 朴实, 简洁, 清新。大量的西岭山歌正是通过简单易唱的旋律, 琅琅上 口的歌词，反映西岭人民生活的各个方面。

如: 呀妹调的代表作品《西岭背二哥》

西岭那个汉子（噢哟嘿）名字（哟）多（哎）, 有一个名字叫（呀）妹儿背二（哟）哥（哎 耶), 背着那个上山（噢哟嘿）忙农（哟）活（耶）, 背粮上山（呀），妹儿去挖（哦）药 
（耶嗬），背那个煤炭（噢哟嘿），又背（哟）木头（耶），跟烟帮夹金山（呀），妹儿还 是当背二（哟）哥（耶哟）。

这是一首描写男女之间爱恋之情的情歌，其中演唱者以 “背二哥” 的爱人的身份，借由对背 二哥工作情况的描述, 表达对 “情哥哥” 的爱慕之意。歌曲旋律简单、平稳、语言生动贴切, 节奏明显, 规律性强。

因此，总的来说，西岭山歌的曲式结构是比较简洁，体现了西岭山歌的纯朴与美丽。

\section{5 结语}

西岭山歌是西岭雪山汉藏羌多民族融合的智慧结晶, 体现的是汉藏㒸各民族人民的生活和情 感, 是我国优秀民族文化的产物, 在我国的非物质文化遗产中占有重要的地位。我们要重视 西岭山歌的传承和保护，找到一条适合西岭山歌传承和发展的路子，这是摆在我们面前的问 题, 需要当地政府、组织、民间艺人和广大人民的不懈努力。只有这样, 西岭山歌这朵汉藏 芫多民族融合的中华民族音乐奇菂，才不会 “断流” ，并且在保护中不断创新和发展，承先 启后，继往开来。

\section{References}

[1]. Sichuan province Daye County Compilation Committee. Dayi County [M]. Chengdu: Sichuan people's publishing house, 1991

[2]. Zhang Dashed. Mountain soul voices: Xiling mountain chants and its legend [M]. Beijing: Popular Literature Press, 2010

[3]. Tang Yu. Qiang Folk Songs' classification and features [J]. Art education, 2012 (07), 98-99.

[4]. Wei Xiao an. A comparative study of the difference of the original ecology of Tibetan folk art features in Sichuan: as an example of dialect area from the Kangba, Ando and Jiarong [J]. Journal of University of Tibet (SOCIAL SCIENCE EDITION), 2016 (01), 107-113.

[5]. Lou Honey, Li Xing. Tibetan folk songs categories and characteristics in Ando, Qinghai [J]. Journal of Qinghai Normal University (PHILOSOPHY AND SOCIAL SCIENCES EDITION), $2012(34 / 6), 104-107$.

[6]. Kuang Tianqi. Classification and category characteristics of Han folk, Sichuan[J]. music exploration: Journal of Sichuan Conservatory of Music, 1984 (3).

[7]. Daye held Xilinx's elderly singing folk contest: caring for the original ecological folk song, let uncle and aunt singing [N]. Chengdu daily, 2006-9-25.

[8]. Du Pin. Dayi's Xiling folk songs has been approved by the Sichuan provincial intangible cultural heritage [N]. Chengdu daily, 2011-10-17.

[9]. Yang Huachun. The mountains as playing stage, heartily singing folk songs [N]. Chengdu daily, 2012-11-26. 